\title{
Role of Surgery in the Elderly Patients Affected from Advanced Stage Ovarian Cancer
}

\author{
E. V. Cafà, B. Pecorino*, G. Scibilia, P. Scollo \\ Division of Obstetrics and Gynecology, Department Maternal and Child, Cannizzaro Hospital, Catania, Italy \\ Email: eliopek@gmail.com
}

Received 11 April 2015; accepted 15 May 2015; published 18 May 2015

Copyright (C) 2015 by authors and Scientific Research Publishing Inc.

This work is licensed under the Creative Commons Attribution International License (CC BY). http://creativecommons.org/licenses/by/4.0/

(c) () Open Access

\section{Abstract}

The aim of this study is to compare morbidity, surgical treatment and post-operative complications in elderly patients underwent surgery for advanced stage ovarian cancer, comparing to younger patients. Data of patients underwent surgery at the Department of Obstetrics and Gynecology of Cannizzaro Hospital (Catania) for advanced stage (IIIC-IV) ovarian cancer were collected from January 2000 to December 2013. Patients were stratified by age in two groups (I > 65 years and II $<65$ years old). Following variables were collected: stage of the tumor, associated diseases, previous chemotherapy, the type of surgical procedures, blood transfusions, intraoperative and postoperative morbidity, mortality, and hospital stay. Median values between the two groups were compared using Mann-Whitney test and frequency data using $\chi^{2}$. Statistical significance was defined as $P<0.05$. A total of 179 patients were identified, they were divided into 2 groups: 64 patients were age 65 years or older (group I) and 115 patients were younger than age 65 (group II). In the whole series, 157 patients $(87 \%)$ did not experience any complication. Overall, postoperative complications occurred in $10(15 \%)$ patients in the group I and in $12(10 \%)$ in the group II ( $p=$ NS). In conclusion, elderly patients may tolerate well surgical procedures within acceptable postoperative morbidity, a length of hospital stay and a need for intensive care quite similar to that of younger patients.

\section{Keywords}

Elderly, Surgery, Ovarian Cancer

\section{Introduction}

Cancer in the older persons has become an increasingly common problem. In Europe, the population aged over

\footnotetext{
"Corresponding author.
}

How to cite this paper: Cafà, E.V., Pecorino, B., Scibilia, G. and Scollo, P. (2015) Role of Surgery in the Elderly Patients Affected from Advanced Stage Ovarian Cancer. Journal of Cancer Therapy, 6, 428-433. 
65 years at present is $13 \%$ and is expected to increase further, with $24.4 \%$ for the year 2050 . Moreover $58 \%$ of all female cancer occurs in women older than 65 years; in particular $43 \%$ of epithelial ovarian cancer is diagnosed in this age group [1]-[3]. However, this population receives substandard treatment when compared to the younger people, generally due to the presence of associated medical conditions. Thus, the gynecologic oncologists will face an increasing proportion of elderly patients in the near future. The treatment for gynaecological malignancies is well defined with respect to the surgery, radiation treatment and chemotherapy. The standard therapy for ovarian cancer is cytoreductive surgery followed by combination chemotherapy [4]. This aggressive surgical approach has an impact for the elderly women, because the prevalence of comorbidity increases with age. In the elderly, the higher incidence of complications and the increased operative mortality often outweigh the uncertain therapeutic results [5]. However, in recent years, new developments in the fields of anesthesiology, perioperative care, and surgical techniques have modified the picture [6].

We investigated a large series of patients, who underwent surgery for advanced stage (IIIC-IV) ovarian cancer at our department in the past decade. We analyzed the clinical features of the patients and the outcome in terms of operative morbidity and mortality, and we compared the same parameters by age of patient (young and elderly) in order to investigate whether clinical features did influence the feasibility and tolerability of surgery in elderly patients.

Overall, the aim of this study is to compare the surgical treatment and post-operative complications between these two groups.

\section{Materials and Methods}

We retrospectively collected all consecutive cases of patients underwent surgery for ovarian cancer at the Department of Obstetrics and Gynecology, Cannizzaro Hospital, from January 2000 to December 2013. Patients were stratified by age in two groups, group I > 65 years and group II < 65 years. We reviewed patients' charts and recorded the following variables: weight, and height, stage of the tumor, associated diseases, previous chemotherapy, type and duration of surgical procedures, preoperative and postoperative hemoglobin count, blood transfusions, intraoperative and postoperative morbidity, mortality, and hospital stay. Before surgery, the anesthetic risk was evaluated by the Standard Physical Status Classification System of the American Society of Anesthesiology (ASA).

In this study, all patients were submitted to explorative laparoscopy/laparotomy in order to evaluate the feasibility of optimal cytoreduction and, based on the intraoperative findings, were routed to continue with surgery or in alternative to start NACT. All types of surgery (debulking surgery, systematic lymphadenectomy, or explorative laparotomy) were considered and recorded. We defined complete cytoreduction the absence of any macroscopic residual tumor (RT) at the end of the procedure; optimal RT consisted of remaining lesions $<1 \mathrm{~cm}$. All patients were treated with platinum-based chemotherapy; 6 adjuvant cycles in patients underwent successful PDS and 3 neoadjuvant cycles in patients routed to IDS followed by 3 adjuvant cycles after successful debulking.

Febrile morbidity was defined as two oral temperatures greater than $38^{\circ} \mathrm{C}$, taken at least $4 \mathrm{~h}$ apart, starting 24 $\mathrm{h}$ following surgery. Severe post-operative complications were recorded. Pulmonary embolism was considered only when ascertained by echo Doppler evaluation. Sepsis was diagnosed only when confirmed by isolation of an infectious agent in the blood. Perioperative mortality was defined as death occurring within $48 \mathrm{~h}$ from the induction of anesthesia. Post-operative mortality was defined as death occurring between $48 \mathrm{~h}$ after the start of anesthesia and 30 days after the last surgical procedure [7]. Wound dehiscence, intestinal occlusion, retroperitoneal abscess were considered too. We calculated patients' distribution by clinical and surgical characteristics and evaluated the results of surgery on the whole series. The median values between the two Groups were compared using the Manne Whitney U test. Frequency data were evaluated by Chi-square test.

Statistical significance was defined as $\mathrm{P}<0.05$.

\section{Results}

A total of 179 patients who met study selection criteria were identified, they were divided into 2 groups: 64 patients were age 65 years or older (group I) and 115 patients were younger than age 65 (group II). The distribution of patients by age, stage, obesity, ASA physical status classes, and presence of associated diseases is presented in Table 1. Overall, 91 (51\%) patients had preexisting medical illnesses, and 45 (25\%) were ASA class 
III-IV. Difference was significative among the two groups. Overall, 97 (54\%) of patients were underwent to major surgical procedures including hysterectomy, salpingo-ovariectomy, total omentectomy and systematic pelvic and lomboaortic nodes dissection. A systematic lymphadenectomy was performed in 28 (43\%) of the elderly persons vs 60 (52\%) in the young. Overall, 22 patients (12\%) were underwent to neoadjuvant chemotherapy and interval debulking surgery successively. Overall, optimal cytoreduction (RT $<1 \mathrm{~cm}$ ) was achieved in 89 (50\%) patients: 29 (45\%) patients of the I group, 60 (52\%) patients of the II group. No significant difference was found among the two groups. In the whole series, 157 patients (87\%) did not experience any complication. Overall, postoperative complications occurred in 10(15\%) patients in the group I and in $12(10 \%)$ in the group II (p = NS). The type and frequency of severe postoperative complications are detailed in Table 2. Two patients in the group I required postoperative intensive care because of respiratory failure and cardiac failure. The median duration of hospital stay was similar between the two groups. In the whole series, no patient died perioperatively.

\section{Discussion}

The increase in life expectancy is combined with a risk of developing a solid tumour. Most cancer-related deaths occur within patients aged 65 years or older, however this expanding population receives substandard treatment when compared to the younger age group [8].

This is due to the perceived limitations to offer conventional treatments to the elderly as there are concerns about associated medical conditions, shorter life expectancy, reluctance to incorporate older patients either into clinical trials of novel agents, or into existing screening programs [9]. Although this concern refers to the overall

\begin{tabular}{lccc} 
Table 1. Patients characteristics. & & & \\
\hline & I group (64 pts) & II group (115 pts) & P \\
\hline AGE (years) & $72(65-94)$ & $51(22-64)$ & \\
STAGE & & & \\
IIIC & $61(95 \%)$ & $105(91 \%)$ & \\
IV & $3(5 \%)$ & $10(9 \%)$ & $<0.05$ \\
Obesity & $10(16 \%)$ & $10(8 \%)$ & $<0.05$ \\
ASA physical status & & & \\
$\bullet$ Class II & $34(53 \%)$ & $100(87 \%)$ & \\
$\bullet$ Class III & $25(39 \%)$ & $13(11 \%)$ & \\
• Class IV & $5(7 \%)$ & $2(2 \%)$ & \\
Patients with comorbidity & $42(65 \%)$ & $38(33 \%)$ & $<0.05$ \\
Optimal cytoreduction $(\mathrm{RT}<1 \mathrm{~cm})$ & $29(45 \%)$ & $60(52 \%)$ & $\mathrm{NS}$ \\
\hline
\end{tabular}

Table 2. Postoperative complications.

\begin{tabular}{cccc}
\hline & I group (64 pts) & II group (115 pts) & P \\
\hline Total complications & $10(15 \%)$ & $12(10 \%)$ & NS \\
Wound dehiscence & $3(4 \%)$ & $4(3 \%)$ & NS \\
Intestinal occlusion & $1(1 \%)$ & $1(1 \%)$ & NS \\
Retroperitoneal abscess & $1(1 \%)$ & $1(1 \%)$ & NS \\
Pulmonary embolism & $2(3 \%)$ & $2(2 \%)$ & NS \\
Deep venous thrombosis & $2(3 \%)$ & $3(3 \%)$ & NS \\
Sepsis & $1(1 \%)$ & $1(1 \%)$ & NS \\
\hline
\end{tabular}


management of the elderly with cancer involving multimodal treatment, surgery is probably the most successful treatment option at the present moment.

A recent retrospective study suggests that elderly patient could be safely managed in the same way of younger patients without an increasing of morbidity and with clinical outcomes similar to those reported in younger case [10].

The increased age of the population is accompanied by an increase of age-related diseases, such as cardiovascular disease, chronic obstructive pulmonary disease, hypertension, arthritis, diabetes mellitus and other malignancies. Beside the tumour stage, with higher stages in elderly cancer patients, the assessment of the comorbidity is an important aspect of the surgical decision-making process [11].

The current standard combined treatment approaches of surgery. chemotherapy and radiation treatment are well defined for the different solid tumours, but there is a need for further exploration in the elderly cancer patients. Improvement in the diagnostic and surgical care of elderly cancer patients will have a final impact on disease-free and overall survival rates of the different types of cancer treatment.

Women with ovarian cancer have a high incidence rate of local recurrences. Although the survival across all stages is $40 \%$, patients with advanced disease have a 5-year survival rate of only $23 \%$. Invasive epithelial ovarian cancer is largely a disease of postmenopausal women, with reproductive age women comprising only $20 \%$ of all patients [6]. Many published studies have specified age as a significant prognostic factor; however, some reports have shown that younger age is not an independent prognostic factor for improved survival [12].

The standard therapy is cytoreductive surgery followed by combination chemotherapy. Studies suggest that those patients with zero residual tumor at conclusion of cytoreductive surgery have significantly longer survival than those patients considered optimal at $0 \mathrm{~cm}$ to $1 \mathrm{~cm}$ [13].

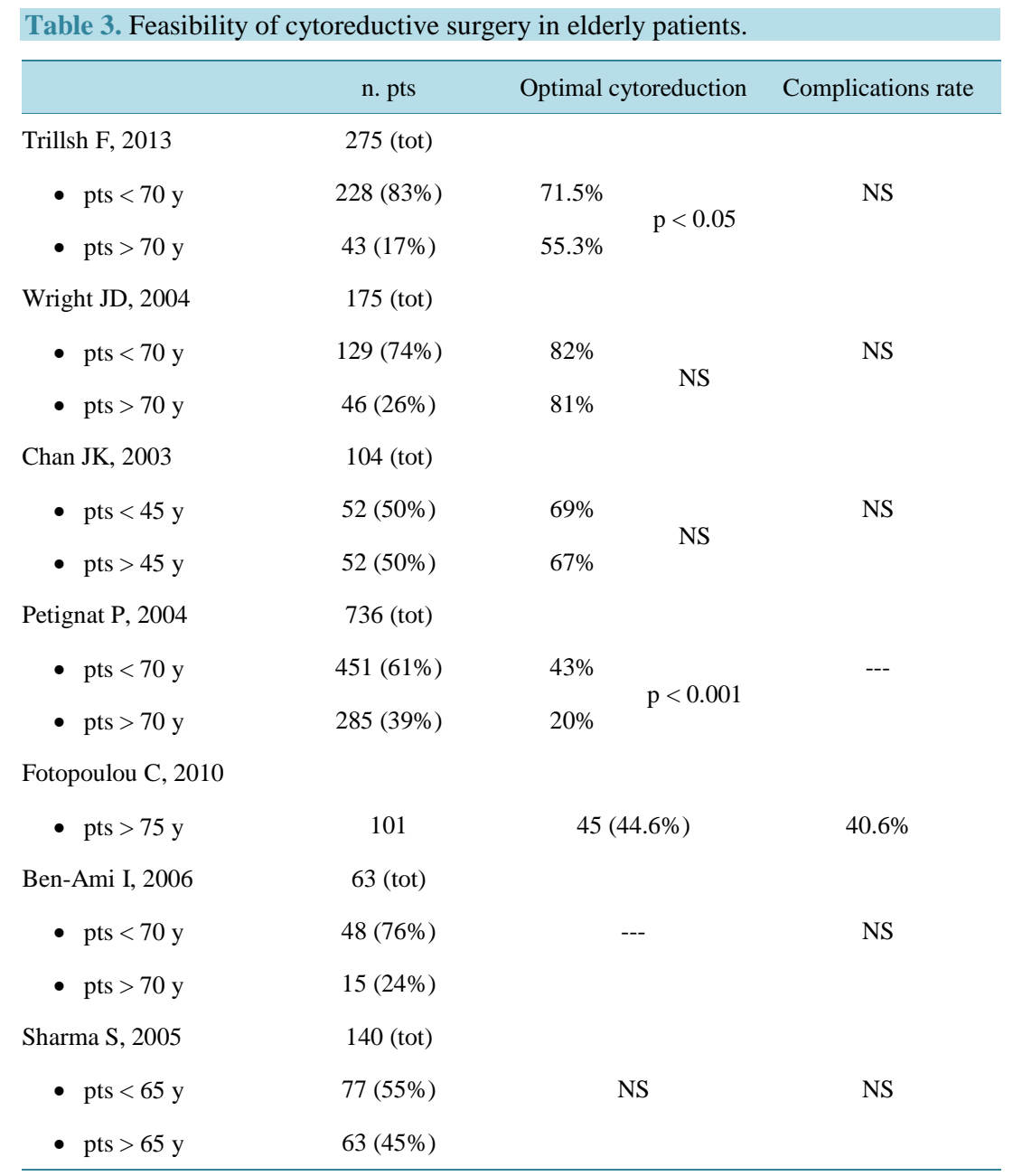


Maximal cytoreductive surgery is one of the most powerful determinants of survival in patients with stage III and IV ovarian cancer. Unfortunately, older patients underwent fewer operations as compared with those of their younger counterparts [14]. It is commonly believed that older patients might not be able to tolerate the debilitating effects of radical surgery. Both cytoreductive surgery and chemotherapy require good physiologic capacities and because elderly patients are more likely to have comorbid conditions as hypertension, cardiac disease, diabetes or others contraindicating these therapeutic approaches, they are less likely to receive optimal surgery and chemotherapy [15]. Several studies have evaluated ovarian surgery in elderly [11] [14] [16]-[18]; they confirm the feasibility of optimal cytoreduction with a complication rate similar to younger patients (Table 3); advanced age should not be considered a contraindication to cytoreductive surgery [14] [19]. In our research, only two recent studies [8] [20] confirm, in their experience, that elderly patients with ovarian cancer are often treated less radically than younger patients. However, our results show that elderly patients may tolerate well surgical procedures within acceptable postoperative morbidity, a length of hospital stay and a need for intensive care quite similar to that of younger patients. The introduction of several expedients in the surgical technique, as well as in the perioperative management, resulted in a significant improvement of surgical rates. It is likely that such expedients, already proven beneficial in series not selected by age are particulary useful in geriatric patients, in whom blood loss reduction and prevention of ischemic, thromboembolic, and infectious disorders may greatly influence the feasibility and tolerability of surgery. We confirm that the majority of elderly patients are able to tolerate the standard of care for ovarian cancer including initial surgical cytoreduction followed by platinum and taxane chemotherapy. For elderly patients with significant medical comorbidity, the extent of surgery and aggressiveness of chemotherapy should be tailored to the extent of disease, symptoms, overall health, and life goals. In addition, enhanced cooperation between geriatricians and oncologists may assist the pretreatment assessment of elderly patients and improve treatment guidelines in this population.

\section{Conclusion}

Our study is limited by the purview of a retrospective study and also incomplete follow-up information to obtain survival analysis. Nevertheless, our data suggest that elderly patients may tolerate well surgical procedures within acceptable postoperative morbidity, a length of hospital stay and a need for intensive care quite similar to that of younger patients.

\section{References}

[1] Yancik R. (1997) Cancer Burden in the Aged: An Epidemiologic and Demographic Overview. Cancer, 80, 1273.

[2] United Nations Department for Economic and Social Information and Policy Analysis. Population Division (1995) World Population Prospects: The 1994 Revision.

[3] Ferlay, J., Black, R.J., Pisani, P., Valdivieso, M.T. and Parkin, D.M. (1996) EUCAN 90: Cancer in the European Union. IARC Cancerbase n. 1, IARC Press, Lyon, France.

[4] The Kelly Gynecologic Oncology Service. The Sidney Kimmel Comprehensive Cancer Center (2007) Primary Cytoreductive Surgery for Advanced Stage Ovarian Cancer: Indications for Radica Resection. Cancerologia 2:1.

[5] Andersen, S.L., Terry, D.F., Wilcox, M.A., Babineau, T., Malek, K. and Perls, T.T. (2005) Cancer in the Oldest Old. Mechanisms of Ageing and Development, 126, 263-267. http://dx.doi.org/10.1016/j.mad.2004.08.019

[6] Susini, T., Scambia, G., Margariti, P.A., et al. (1999) Gynecologic Oncologic Surgery in the Elderly: A Retrospective Analysis of 213 Patients. Gynecologic Oncology, 75, 437-443. http://dx.doi.org/10.1006/gyno.1999.5591

[7] Chassagne, D., Sismondi, P., Sinistrero, G., et al. (1993) A Glossary of Reporting Complications of Treatment in Gynecological Cancers. Radiotherapy and Oncology, 26, 195-202. http://dx.doi.org/10.1016/0167-8140(93)90260-F

[8] Petignat, P., Fioretta, G., Verkooijen, H.M., et al. (2004) Poorer Survival of Elderly Patients with Ovarian in Cancer: A Population-Based Study. Surgical Oncology, 13, 181-186. http://dx.doi.org/10.1016/j.suronc.2004.08.010

[9] Audisio, R.A. (2004) The Surgical Risk of Elderly Patients with Cancer. Surgical Oncology, 13, 169-173. http://dx.doi.org/10.1016/j.suronc.2004.09.012

[10] Fanfani, F., Fagotti, A., Salerno, M.G., Margariti, P.A., Gagliardi, M.L., Gallotta, V., Vizzielli, G., Panico, G., Monterossi, G. and Scambia, G. (2012) Elderly and Very Elderly Advanced Ovarian Cancer Patients: Does the Age Influence the Surgical Management? European Journal of Surgical Oncology, 38, 1204-1210.

[11] Chan, J.K., Loizzi, V., Lin, Y.G., Osann, K., Brewster, W.R. and DiSaia, P.J. (2003) Stages III and IV Invasive Epithelial Ovarian Carcinoma in Younger versus Older Women: What Prognostic Factors Are Important? Obstetrics \& Gy- 
necology, 102, 156-161. http://dx.doi.org/10.1016/S0029-7844(03)00399-5

[12] Geisler, J.P. and Geisler, H.E. (2001) Radical Hysterectomy in the Elderly Female: A Comparison to Patients Age 50 or Younger. Gynecologic Oncology, 80, 258-261. http://dx.doi.org/10.1006/gyno.2000.6044

[13] Langstraat, C., Aletti, G.D. and Cliby, W.A. (2011) Morbidity, Mortality and Overall Survival in Elderly Women Undergoing Primary Surgical Debulking for Ovarian Cancer: A Delicate Balance Requiring Individualization. Gynecologic Oncology, 123, 187-191. http://dx.doi.org/10.1016/j.ygyno.2011.06.031

[14] Pignata, S. and Vermorken, J.B. (2004) Ovarian Cancer in the Elderly. Critical Reviews in Oncology/Hematology, 49, 77-86. http://dx.doi.org/10.1016/S1040-8428(03)00100-8

[15] Wright, J.D., Herzog, T.J. and Powell, M.A. (2004) Morbidity of Cytoreductive Surgery in the Elderly. American Journal of Obstetrics and Gynecology, 190, 1398-1400. http://dx.doi.org/10.1016/j.ajog.2004.01.078

[16] Christina, F., Konstantinos, F., Elisabeth, S.-T., Marcus, B., Werner, L. and Jalid, S. (2010) Primary Radical Surgery in Elderly Patients with Epithelial Ovarian Cancer: Analysis of Surgical Outcome and Long-Term Survival. International Journal of Gynecological Cancer, 20, 34-40. http://dx.doi.org/10.1111/IGC.0b013e3181c10c04

[17] Ben-Ami, I., Vaknin, Z., Schneider, D. and Halperin, R. (2006) Perioperative Morbidity and Mortality of Gynecological Oncologic Surgery in Elderly Women. International Journal of Gynecological Cancer, 16, 452-427. http://dx.doi.org/10.1111/j.1525-1438.2006.00478.x

[18] Sharma, S., Driscoll, D., Odunsi, K., Venkatadri, A. and Lele, S. (2005) Safety and Efficacy of Cytoreductive Surgery for Epithelial Ovarian Cancer in Elderly and High-Risk Surgical Patients. American Journal of Obstetrics and Gynecology, 193, 2077-2082. http://dx.doi.org/10.1016/j.ajog.2005.06.074

[19] Cloven, N.G., Manetta, A., Berman, M.L., Kohler, M.F. and DiSaia, P.J. (1999) Management of Ovarian Cancer in Patients Older Than 80 Years of Age. Gynecologic Oncology, 73, 137-139. http://dx.doi.org/10.1006/gyno.1998.5337

[20] Trillsch, F., Woelber, L., Eulenburg, C., et al. (2013) Treatment Reality in Elderly Patients with Advanced Ovarian Cancer: A Prospective Analysis of the OVCAD Consortium. Journal of Ovarian Research, 6, 42. http://dx.doi.org/10.1186/1757-2215-6-42 